Selcuk Journal of Agriculture and Food Sciences

http://sjafs.selcuk.edu.tr/sjafs/index

Review Article
SJAFS

(2020) 34 (2), 169-177

e-ISSN: $2458-8377$

DOI:10.15316/SJAFS.2020.212

\title{
Doubled Haploid Production in Cereals Using Microspore Culture
}

\author{
(D) Neslihan Doruk ${ }^{1, *}$, (D) Nejdet KANDEMİR ${ }^{2}$, (DSSabri GÖKMEN ${ }^{1}$ \\ ${ }^{1}$ Selçuk University, Faculty of Agriculture, Department of Field Crops, Konya, Turkey \\ ${ }^{2}$ Tokat Gaziosmanpaşa University, Faculty of Agriculture, Department of Field Crops, Tokat, Turkey
}

\begin{tabular}{l}
\hline ARTICLE INFO \\
\hline Article history: \\
Received date: 04.05 .2020 \\
Accepted date: 25.05 .2020 \\
\hline Edited by: \\
Ali KAHRAMAN; Selçuk University, \\
Turkey \\
Reviewed by: \\
Ertan SAit KURTAR; Selçuk University, \\
Turkey \\
Selçuk KODAZ; Atatürk University, \\
Turkey \\
Mustafa YILDIZ; Ankara University, \\
Turkey
\end{tabular}

Keywords:

Albino plant

Co-cultivation

Embryo induction

Green plant

Microspore isolation

Pretreatment

\begin{abstract}
Doubled haploids are extremely useful in plant breeding since they provide rapid homozygosity. However, the success rate of doubled haploid production in cereals is still not high enough, and there is a special problem involving the formation of high percentage of albino plants. Nevertheless, the success rate in microspore culture in cereals is higher than classical anther cultures, and the method has the advantage of spontaneous chromosome doubling. On the other hand, this method has some critical stages such as pretreatments and microspore isolation, and these stages need to be optimized for the successful use of the technique in plant breeding. For this aim, there have been studies in recent years about combining the pretreatment practices, supplementing growth media with a variety of ingredients, improving the various co-culturing practices and decreasing the albino plant percentage. This technique has been commonly used in the world especially in barley and wheat breeding. Improving the success rate of the technique will be useful for its integration into modern breeding techniques such as apomictic crops and transgenics.
\end{abstract}

\section{Introduction}

Haploids are plants that have half the number of chromosomes that a species normally has. These plants are mostly sterile since they can't form gametes. In order to avoid the sterility, doubled haploid plants are produced from haploids through doubling their chromosome numbers using various methods. These plants attaining homozygosity in just one generation have potential uses in plant breeding. However, their low production efficiency is a major obstacle for their common use in plant breeding (Devaux and Kasha 2009).

Haploid plants can naturally occur with apomixis mechanisms such as parthenogenesis and semigamy (Bashaw 1980). Artificially, they can be produced using relatively traditional techniques such as anther culture (Germana 2011), ovule or ovary cultures (Chen et al. 2011), chromosome elimination (Houben et al. 2011) and irradiated pollen (Sestili and Ficcadenti 1996) and using advanced methods such as microspore

\footnotetext{
*Corresponding author email: neslihan.doruk@selcuk.edu.tr
}

culture (Ferrie and Caswell 2011) and centromere engineering (Tek et al. 2015). The method of choice largely depends, among other factors, on the degree of success rates in the relevant crop species.

One of the methods that have been heavily researched in recent years for the production of doubled haploid plants in cereals is the microspore culture (Esteves and Belzile 2019; Wang et al. 2019; Zur et al. 2019). Being a more sophisticated method, microspore culture has a higher success rate since the impact of the pretreatment methods on the microspores used to divert them from the gametophytic to sporophytic pathway is direct without the protective tissues of the anther. Compared to anther culture, microspore culture was reported to increase plant regeneration by 5-200 times (Hoekstra et al. 1992; Davies and Morton 1998). The method also has additional advantages such as producing only haploid plants and having the ability of spontaneous chromosome doubling (Li and Devaux 2001). However, this method has many critical procedures compared to anther culture, and optimizing them is a necessity for successful use of the method in plant breeding. 
Microspore culture, one of the methods used to achieve rapid homozygosity in plant breeding, has strong potential to increase the effectiveness of plant breeding (Kasha et al. 2003; Li and Devaux 2001). The successful use of this method could significantly accelerate the breeding of pureline and hybrid varieties. However, such an effect can only be achieved by elaboration of many stages involved with this method. The aim of the present review is to introduce various critical stages of the microspore culture method and to discuss the ways to improve them with the current research.

\section{Use of haploids or doubled haploids}

Doubled haploid plants are $100 \%$ homozygous for all loci, and are very useful for plant breeding purposes. They could be used for rapid production of truebreeding lines from the segregating progeny of crosses, inbred lines in hybrid variety programs and doubled haploid mapping populations for quantitative trait mapping purposes (Rajcan et al. 2011). Homozygosity, traditionally achieved in 6-8 generations, is achieved in only one generation with doubled haploidy method (Jahne and Lörz 1994). Doubled haploids have been increasingly used in breeding of cereal species. For example, it has been estimated that about half of the registered barley varieties in Europe has been produced using doubled haploidy (Forster et al. 2007).

The haploidy or doubled haploidy method is used to produce interspecific hybrids for plant breeding. Cultivated potatoes with tetraploid genome composition produce sterile triploids when crossed to diploid wild species for an aim of transferring useful genes from them. However, when the chromosome number of potatoes is reduced to diploid level using haploidy method, resulting diploid plants could be easily crossed with diploid wild relatives and produce fertile seeds (Jansky 2006). The chromosome number of these diploid interspecific hybrids could be doubled to restore the original tetraploid genome of the cultivated potato. Thus, valuable genes could be introgressed to other potato varieties in future crosses with potato cultivated varieties.

Doubled haploid mapping populations from biparental crosses are commonly used for mapping the quantitative traits. Since they have all loci in homozygous conditions, their genetic analyses are more robust. Besides, since they could be multiplied indefinitely, doubled haploid lines allow producing seeds enough to plant rows or even plots to observe quantitative traits that cannot be observed in single plants such as lodging or grain yield. Thus, it could even be possible to conduct multilocational trials for more complex traits (Humphreys and Knox 2015).

Mutation or transgenic studies carried on haploid materials have the advantage of producing homozygous material. Thus, no extra generation is needed to convert heterozygous (mutants) or hemizygous (transgenics) plants into true breeding homozygous lines to observe the effects of the genetic changes created (Shariatpanahi and Ahmadi 2016).

\section{Microspore development in plants}

Microspore mother cells are formed in pollen sacs of the anthers. These cells with diploid (2n) chromosome sets undergo meiosis and give rise to four daughter cells with haploid (n) chromosomes called microspore (uninucleate stage). Then, each microspore nucleus (n) undergoes mitosis and forms pollen grain with two nuclei. For a great majority of the plants, the best microspore developmental stage for production of embryos (i.e. androgenesis) was reported to be mid-late uninucleate stage (Zheng 2003). Determination of the developmental stage of the microspores and using the ones in correct stage is crucial for the success of the microspore cultures. Nuclei in the microspores are observed to determine their developmental stages.

Appearance of plants at the best stage for microspore culture could change by the genotype. In barley, this is a stage at a point from the extension of the last internode between the flag leaf and penultimate leaf to the awn appearance, and is mostly when this internode is $2 \mathrm{~cm}$. However, this appearance could vary by the growing conditions of donor plants such as fields, greenhouses or growth chambers. Therefore, it is a necessity to observe the best stage of microspores before conducting microspore culture for a set of plants. After such a determination, the plants can be judged based on their appearance only. For such an observation, anthers from the middle of the spikes of a series of plants with consecutive developmental stages (Figure 1) are extracted and squashed in an eppendorf tube containing $2 \%$ acetocarmine solution using a pipet tip. After a two-hour incubation period in acetocarmine, microspores are observed under a microscope. In uninucleate stage, only the nucleus is stained, and the remaining parts of the microspore are relatively clear. On binucleate stage, the second nucleus is generally diffused and rarely observed as a compact structure. However, this stage is easily distinguished by the staining of all microspore due to the starch accumulated (Figures 2 and 3). 


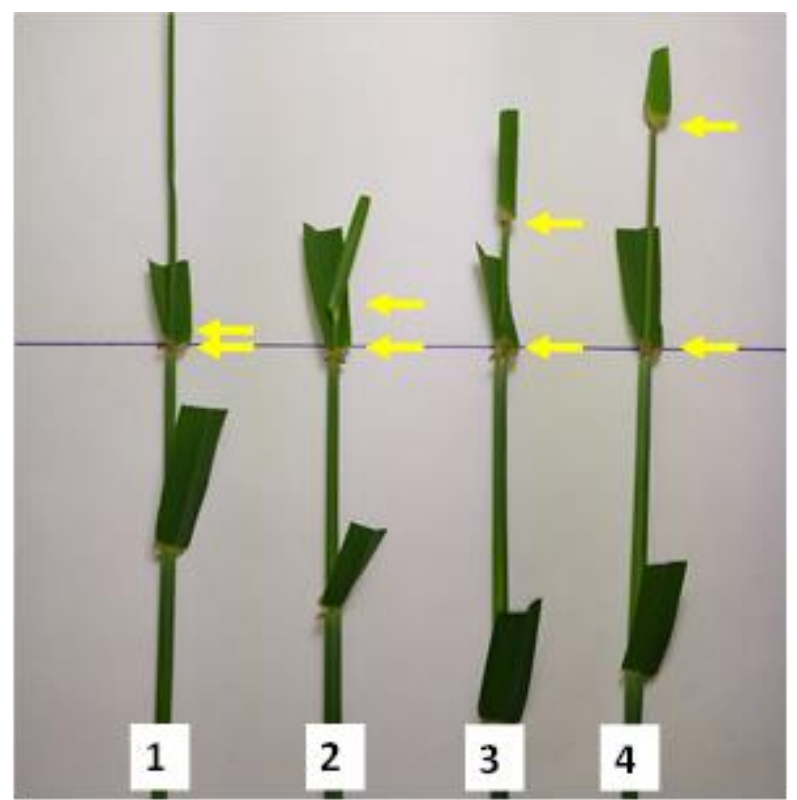

Figure 1

Determining the appropriate stage of spike development for microspore culture. After examination of acetocarmine-stained microspores, the best stage in which most of the microspores were at mid-late uninucleate stage was found to be 3 .

\section{Microspore culture procedure}

Microspore culture is the method of isolating and culturing immature microspores on special media un-

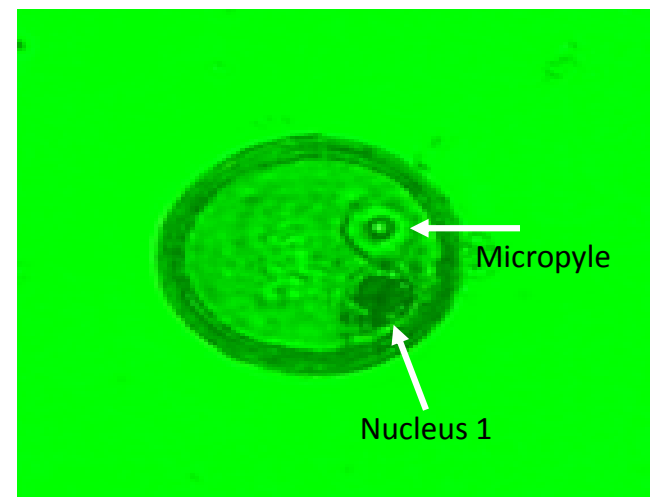

Figure 2

Late uninucleate stage when the single nucleus is close to microphyll opening and the rest of the microspore is not well stained. This stage is appropriate for microspore culture

\subsection{Growing conditions of donor plants}

Stimulating the microspores to induce embryos in vitro is highly dependent on the growing conditions of the plants from which the microspores are obtained. Donor plants need to be strong, healthy and devoid of all kinds of stress conditions. Environmental factors such as water status, diseases, temperature, moisture, aeration, photoperiod, light quality and intensity and plant nutrition during the period in which plants are der aseptic conditions to produce plants with haploid chromosome numbers (Chen et al. 2011). It is a more elaborate procedure compared to anther culture and involves many critical stages. Among them are isolation and purification of microspores and determination of their number and viability. The success rate in microspore culture is dictated by factors such as genotype, growing conditions of donor plants, microspore isolation methods, pretreatments, culture media and growing conditions in tissue culture.

\subsection{Genotype}

As in all tissue culture procedures, genotype is a major determinant of the success of microspore culture. First of all, the responses of plant species to microspore culture are different, which could be extremely low in some species (Soriano et al. 2013). In general, androgenic response is not high in cereal crop species. Although the efficiency of doubled haploidy could be sufficient for plant breeding purposes in barley and wheat, success rate is not satisfactory with oat and rice (Ferrie and Caswell 2011). The androgenic response could also vary at genotype level within the species. Two-row barley genotypes were reported to have better response to microspore culture compared to six-row cultivars. Two-row winter barley cultivar Igri is used as standard in microspore culture studies of barley (Davies 2003). In wheat, on the other hand, cvs. Chris, Pavon 79 and Bob White are well known for their superior androgenic response (Kasha et al. 2003).

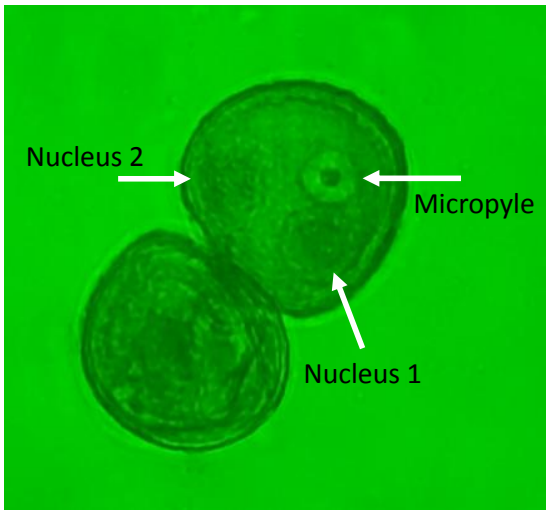

Figure 3

Binucleate stage where microspore is thoroughly stained due to starch accumulation. This stage is late for microspore culture.

grown can have an impact on the success of microspores obtained from those plants.

Donor plants for microspore culture can be grown in fields, greenhouses and growth chambers. Field conditions provide strong plants with high androgenic response compared to greenhouse grown plants (Ouyang et al. 1993), but they have a higher risk of contamination in tissue culture. Growth chambers are places with strictly controlled environmental conditions. However, due to their limited space, they may not be suitable for large scale studies. Climate- 
controlled greenhouse conditions are generally places preferred for growing the donor plants.

Water stress during the growing period of donor plants could lead to smaller plants and microspores. Success rate for haploid plant production is low with this kind of small microspores (Ferrie and Caswell 2011). Similarly, plants should not be overwatered during their growth period.

Necessary precautions should be taken to prevent plants from getting sick and to protect them from pests. When diseases or pest infestations are encountered, the problem should be remedied as early as possible using appropriate chemicals.

Temperature preferences of cereal species during the growth periods vary considerably. When the donor plants of winter barley genotypes are grown at 15/12 ${ }^{\circ} \mathrm{C}$ day/night temperatures, their microspores give the best androgenic response, while $18 / 15^{\circ} \mathrm{C}$ temperatures are better for spring barley cultivars (Kasha et al. 2003). For growing donor wheat plants, $18 / 15{ }^{\circ} \mathrm{C}$ day/night temperatures are optimal ( $\mathrm{Hu}$ et al. 1995), whereas $24 / 20{ }^{\circ} \mathrm{C}$ are best for maize (Gaillard et al. 1991). A photoperiod regime of 16 hours light and 8 hours dark is appropriate for most species. The relative humidity in plant growing area should be about 70$80 \%$ (Jacquard et al. 2003). Optimal light density to grow donor plants for cereal microspore culture was reported to be about 18,000-20,000 lux (ForoughiWehr et al. 1976).

Under greenhouse conditions, plants should be grown in pots no smaller than 3-4 liters. A growing mix of soil, manure, perlite and peat is suitable. Some sand could be added to the mixture if the soil texture is heavy. The pots should be fertilized with nitrogen during or before planting the seeds. However, in order to prevent plants to suffer from nutrient deficiency, they should be fertilized weekly or fortnightly using fertilizers which have micronutrients or nutrient solutions such as Hoagland's.

\subsection{Pretreatments}

Microspores need to be exposed to some pretreatments in order to switch them from the pathway they are programmed for, which is the development of functional pollen, to another pathway of producing embryos. A number of different pretreatments could be employed to do this. Among them are cold, heat shock, starvation and high osmotic pressure treatments, various chemical pretreatments and their combinations (Oleszczuk et al. 2006). These pretreatments could be applied to tillers, spikes, anthers or microspores.

Cold pretreatment: In this pretreatment method, tillers carrying the spikes are kept at $4{ }^{\circ} \mathrm{C}$ for 28 days (Esteves and Belzile 2014a). After the pretreatment period, spikes are taken from the tillers and surface sterilized (one minute at $70 \%$ alcohol, five minutes at $1 \%$ sodium hypochlorite solution containing a few drops of Tween20 , followed by four rinses with sterile distilled water). Then, microspores are isolated. Cold pretreatment was reported to produce 35.3 green plants per spike used in barley, but a high percentage of albino plants were observed (Esteves and Belzile 2014a). Cold pretreatment was found to increase androgenic response in other cereals such as maize, wheat and rice, and was commonly used in microspore cultures of these plants (Oleszczuk et al. 2006).

High osmotic pressure: Spikes are stored in a chemical solution such as mannitol which exerts high osmotic pressure. For this aim, the spikes at the appropriate microspore developmental stage are surface sterilized, and immersed in a $0.3 \mathrm{M}$ sterile mannitol solution. Asif et al. (2014) reported that the production of embryolike structures and green plants were significantly higher in $9.1 \mathrm{~g} / \mathrm{l}$ mannitol pretreatment compared to the control. Besides, green/albino plant ratio was also higher with this pretreatment. Mannitol pretreatment was also found useful in microspore cultures of rice (Raina and Ifran 1998), oats (Ferrie et al. 2014), rye (Zieliński et al. 2020) and triticale (Gland-Zwerger et al. 1994). It is common to combine mannitol pretreatment with heat shock or cold pretreatment methods.

Heat shock pretreatment: Spikes exposed to heat shock of about $26-33{ }^{\circ} \mathrm{C}$ have better androgenic response. Heat shock pretreatment is usually applied after a cold pretreatment or in combination with other pretreatment methods such as high osmotic pressure, starvation or use of various chemicals (Oleszczuk et al. 2006). Liu et al. (2002) reported that success rates of chemical pretreatment methods were better when they were combined with heat shock pretreatment of $33{ }^{\circ} \mathrm{C}$. In barley, green plant production rate of 35.3 per spike increased to 65.4 when the mannitol pretreatment was combined with a 48-hour heat shock treatment at 26 ${ }^{\circ} \mathrm{C}$, and it was mentioned that this increase was due to decreased albino plant percentage (Esteves and Belzile 2014a).

Starvation: Using media lacking nitrogen or sugars increases the androgenic response of the microspores. This treatment is also commonly used together with cold or high osmotic pressure pretreatment methods. This method was reported to be effective in recalcitrant genotypes (Oleszczuk et al. 2006).

Chemical pretreatments: It was reported that treating isolated microspores with some chemicals for short periods (38-52 hours) at temperatures around $25{ }^{\circ} \mathrm{C}$ increases the androgenic response of microspores. Colchicine is one of these chemicals successfully used to increase haploid plant production, which also doubles the chromosomes of the plants produced (Zhao et al. 1996). Zheng et al. (2001), on the other hand, used 10 different chemicals, including 2HNA, and reported that nine of them increased the microspore viability 1.5-2-fold, and produced almost twice as many green plants. Pechan and Keller (1989), on the other hand, stored rapeseed inflorescences in $15 \%$ ethanol for 2-4 days as a pretreatment, and observed that embryo production in microspore culture increased almost twice.

\subsection{Microspore isolation methods}


Microspore isolation is extracting the microspores from the anthers at the correct stage and culturing on growth media. Various methods could be employed to isolate the microspores. Some of them use blenders to homogenize spikes, while some macerate the anthers with a pestle, and some collect the microspores spontaneously shed into a storage solution. In blender method, the spikes are placed in blender's chamber along with about $20-25 \mathrm{ml}$ cold mannitol solution (0.3 M), and blender is run at a low speed for 15-25 seconds. Blender chamber is washed with mannitol and the remaining microspores are collected. In maceration method, anthers are squashed using a glass or Teflon rod after pretreatment (Olsen 1991). In shedmicrospore method, anthers are stored in a solution in petri dishes generally in combination with a heat shock pretreatment $\left(25-32{ }^{\circ} \mathrm{C}\right)$, and they are spontaneously shed into the solution after storing for two-three days (Ziauddin et al. 1990). Vortex, sonication or magnetic stirrer could be used in all these methods for a better isolation of all microspores from the anthers.

The resulting slurry after using above-mentioned methods to release the microspores into a solution contains both microspores and spike pieces, and is passed through $100 \mu \mathrm{m}$ nylon sieves. Microspores passing through the sieve and separated from plant debris is collected using centrifugation performed at about $100 \mathrm{RCF}$ (relative centrifugal force) for four or five minutes. Microspore pellet is cleaned through resuspensions in $0.3 \mathrm{M}$ mannitol for three or four times. Then, microspores are suspended in $21-23 \%$ maltose solution, and empty, dead or broken microspores are removed through a density gradient centrifugation at about $100 \mathrm{RCF}$ for three to five minutes. Finally, microspores are suspended in $200 \mu \mathrm{l}$ liquid induction medium. Microspore concentration is determined using a hemocytometer or by counting all microspores in a small volume (e.g. $20 \mu \mathrm{l}$ ), and the density is adjusted to $10^{6} \mathrm{microspore} / \mathrm{ml}$ using liquid induction medium.

Quality and viability of the microspores need to be checked after the isolation. They could be small, empty or damaged during the isolation process. Sustaining the microspore viability is one of the critical factors affecting the success of the culture. There are some methods to check the quality of microspores. The most common ones are Tetrazolium test (TTC) and acetocarmine staining. Besides, iodine-potassium iodide and aniline blue methods could also be used.

In acetocarmine staining, a small amount of microspore preparation is added to a $2 \%$ acetocarmine solution and 30 minutes later microspores are observed under microscope (Malayeri et al. 2012). Living microspores are stained in red while dead or empty ones are not (Figure 4).

For tetrazolium test, $1 \%$ triphenyl tetrazolium chloride (TTC) dissolved in 5\% sucrose is used. TTC solution is dropped on a microscope slide and a cover glass is put on it. Microspores are observed under a light microscope. Living microspores are stained in red while others remain colorless (Vizintin and Bohanec 2004).

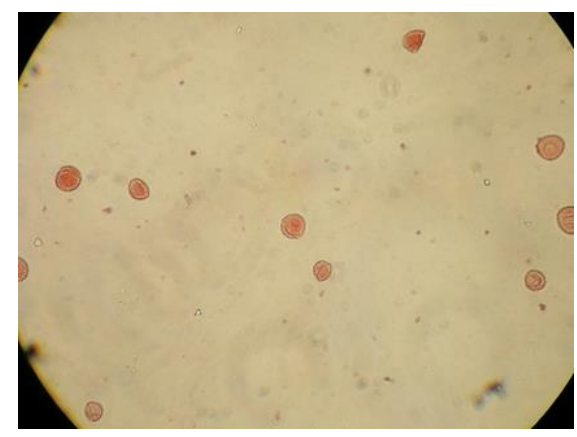

Figure 4

Acetocarmine-stained living microspores

\subsection{Culturing the isolated microspores}

Isolated microspore culture is basically a cell culture method, and its management in tissue culture is more challenging than other commonly used tissue culture explants. Microspores cannot survive on solid media, whereas they are submerged and cannot get enough oxygen in liquid media. In case of culturing in liquid medium, the cultures need to be aerated through continuous shaking, which necessitates an intensive use of shaker equipment, which may not be available in all laboratories. Instead, microspores are commonly cultured as small droplets placed directly on the bottom of petri dishes or on a solid media in a two-layer system (Jahne et al. 1994). A small amount of liquid medium (about $1 \mathrm{ml}$ ) containing microspores could also be spread on a $3 \mathrm{ml}$ solid medium in a $5 \mathrm{~cm}$ petri dish. The density of the microspores is important for the success of the culture. About 100.000 microspores are cultured on a $5 \mathrm{ml}$ petri dish (Esteves and Belzile 2014a).

\subsection{Microspore growth media}

Two types of growth media are used in microspore cultures: first, induction medium in which microspores are induced to produce somatic embryos, and then regeneration medium which is used to produce green plants from somatic embryos.

\section{Induction medium}

The induction medium is basically a modified $\mathrm{Mu}$ rashige Skoog basal medium containing low amounts of nitrogen $(165 \mathrm{mg}$ ammonium nitrate rather than $1,650 \mathrm{mg}$ in regular MS medium) and $60 \mathrm{~g}$ maltose. The induction medium could include $32 \mathrm{~g} / \mathrm{l}$ mannitol. In a two-layer induction medium system, the solid medium in the bottom is solidified using an agar, while the liquid medium containing the microspores does not include any solidifier. A cytokinin (e.g. $1 \mathrm{mg} / \mathrm{l} \mathrm{BAP}$ or TDZ) and a small amount of auxin (e.g. $0.3 \mathrm{mg} / \mathrm{l}$ dicamba) are added to the induction medium (Esteves and Belzile 2014a). Besides, in order to help the viability of microspores in the culture, induction media are supplemented with $50 \mathrm{mg} / \mathrm{l}$ gum arabic and arabinogalactan proteins (Letarte et al. 2006). Microspores cul- 
tured on induction medium are incubated under dark conditions at $28{ }^{\circ} \mathrm{C}$ for 20-30 days (Asif et al. 2014). Developing embryo-like structures (ELSs) are kept under light conditions (16 hours light in 24 hours, with a light intensity of $80 \mu \mathrm{mol} \mathrm{m} \mathrm{m}^{-2} \mathrm{~s}^{-1}$ ) for two or three days. ELSs which show green segments are transferred to regeneration medium.

\section{Regeneration medium}

Regeneration media used in microspore culture are basically modified MS media, and have the same salts, vitamins and organic compounds as the induction media. They may have gum arabic and arabinogalactan proteins, but they do not have mannitol. They include $30 \mathrm{~g}$ maltose instead of $60 \mathrm{~g} / \mathrm{l}$, and have different hormone content. Regeneration media generally contain low levels of cytokinin (e.g. $0.2 \mathrm{mg} / \mathrm{l} \mathrm{BAP}$ or $0.1 \mathrm{mg} / \mathrm{l}$ metatopoline) and auxin (e.g. $0.1 \mathrm{mg} / \mathrm{l} \mathrm{IAA})$ (Esteves and Belzile 2014b). ELSs developing in regeneration media is kept in growth chambers under light conditions. Two weeks after transferring to regeneration media, the number of green sectors in each petri dish is counted, and they are transferred to germination media, which are basically the same as the regeneration media except that they do not include any hormones. Two weeks later, green and albino plants are counted. This method could produce more than 100 green plants per spike in barley (Esteves and Belzile 2014b) and about 90 in wheat (Wang et al. 2019).

\subsection{Co-cultivation}

Although its exact mechanism is not known, cocultivation of cereal microspores along with various flower parts were reported to increase the androgenic response of microspores (Oleszczuk et al. 2006). It was hypothesized that this effect could be due to hormonelike substances released from the ovaries (Köhler and Wenzel 1985) or due to small molecules released from immature pistils (Lipman et al. 2015). The whole floret, ovary or pistil could be used for co-cultivation. There is even a report that mentions the failure of wheat microspores to undergo cell division to produce ELSs without ovary co-cultivation (Patel et al. 2004). Co-cultivation with ovary was reported to cause 2.1and 2.4-fold increases in ELS and green plant productions, respectively, in a recalcitrant barley cultivar $(\mathrm{Li}$ and Devaux 2001). In addition, co-cultivation of barley microspores with pistils cut and placed in microspore medium was reported to cause a five-fold increase in the number of embryogenic calli (Lipman et al. 2015). However, pistils needed to be changed in every four days. Microspores themselves in culture are known to exert a "self-feeder effect" and increase the androgenic response. Lipman et al. (2015) reported that when the microspore density was less than 1000 per milliliter medium, no embryogenic callus was produced.

\subsection{Albinism problem in cereal microspore cultures}

One of the major challenges with cereal microspore cultures is the formation of albino plants. Previously, albinism was thought to be due to the breakdown of plastid DNA (Caredda et al. 2000). However, now it has been understood that inactivation of the plastid ribosome is the culprit (Torp and Andersen 2009).

There are several factors which play roles in albinism in cereal cultures. One of them is the genotype. Durum wheats are known to have a higher degree of albinism (Zheng 2003). Differences were also reported among bread wheat cultivars (Broughton 2008). It was found that culturing microspores on solid media increases the albino plant percentage (Zheng 2003). The position of the spike in the plant is another factor affecting the albinism, and it was reported that the highest green plant percentage was obtained from the second tiller, which was the third spike on the plant after the main shoot and the first tiller (Jacquard et al. 2006). Embryo inductions under light conditions were reported to result in the formation of more than $90 \%$ albino plants (Ziegler et al. 1990). Microspore isolation method was mentioned to be one of the factors affecting albino plant percentage, and isolation using a glass rod and homogenizer caused significant decreases in albino plant production compared to microspore isolation carried out using blenders (Islam et al. 2013). Finally, pretreatment methods affect albinism considerably, and albino plants are more common in cold pretreatment (Oleszczuk et al. 2006).

In eliminating the albinism problem, modifying the pretreatment method seems to be the most feasible one. Use of a 48 -hour heat shock at $26{ }^{\circ} \mathrm{C}$ instead of cold pretreatment reduced the albino plant production prominently (Esteves and Belzile 2014a). Makowska et al. (2017) reported that increasing the copper content of growing medium by 100 folds resulted in a higher number of total plant regeneration and lower percentage of albino plants. Wojnarowiez et al. (2004) found that use of osmotic stress agents decreased albino plant percentage, but there were differences among different osmotic stress agents such as sorbitol, mannitol, sucrose and PEG. Growth regulator composition of the medium could also be modified to alleviate the albinism problem. BAA + IAA combination was reported to be associated with production of fewer albino plants compared to Kinetin + 2,4-D combination in bread wheat (Broughton 2008).

\subsection{Chromosome doubling}

Haploid plants are sterile. Their chromosome numbers need to be restored to the regular chromosome number of the species to be used in plant breeding. In microspore culture, chromosome doubling mostly occurs spontaneously. It was reported that $75-85 \%$ of the plants produced in wheat or barley microspore cultures were spontaneously doubled. Cold pretreatments commonly used in cereal microspores were mentioned as the origin of spontaneous chromosome doubling (Oleszczuk et al. 2006). Spontaneous chromosome doubling is relatively less common in triticale (10-30\%) and maize (4.5-6.5\%) microspore cultures.

When the microspore culture-derived plants are haploids, their chromosomes can be doubled through 
applying colchicine to their growing points. Colchicine prevents the formation of microtubules and chromosomes are not pulled to opposite poles. Thus, ploidy level increases. Ploidy levels of the plants could be determined by physical appearance of the plants, number of stomata underneath the leaves, cytogenetic observations, hemocytometer counting or DNA markers. A commonly used method is cytogenetic observation with a chromosome dye such as acetocarmine carried out on cells of root tips whose cell division is stopped at metaphase. Another robust method is counting the stomata since haploid plants have a higher number of smaller stomata compared to diploid plants.

\subsection{Acclimatization}

Plants produced in microspore culture are transferred to soil when they have enough roots to support them. However, they will die immediately when directly transferred to soil because they do not have cuticular wax on their leaves which protects them against the water loss and their capillary roots are not well developed yet. They need to be acclimatized first. For this aim, plantlets from the microspore culture are transplanted to a few $\mathrm{cm}$ thick water-saturated peat in a pot. Water is sprayed on them, and the top of the pot is sealed using a stretch film tightened with rubber bands. Three to four holes are made on the stretch film on the following day, and these holes are expanded during the next two or three days. Finally, acclimatized seedlings are transplanted to the pots where they will be grown to maturity.

In conclusion, microspore culture has a higher success rate for haploid plant production compared to anther culture. In anther culture, microspores could be protected by the surrounding anther tissues against the external shock of the pretreatments used to divert them into embryo formation. In microspore culture, however, microspores are directly exposed to such effects. Microspore culture also has the advantage of spontaneous chromosome doubling. The success rate for haploid plant production is still not satisfactory in some cereal species especially due to albino plant formation, and this problem should be remedied for an efficient use of the method in plant breeding programs. It is especially important to improve pretreatment methods and growth media compositions to sustain the viability of microspores in culture. Microspore cultures with better success rates would contribute to the modern plant breeding technologies such as apomixis and transgenics.

\section{References}

Asif M, Eudes M, Randhawa H, Amundsen E and Spaner D (2014). Induction medium osmolality improves microspore embryogenesis in wheat and triticale. In Vitro Cellular and Developmental Biology - Plant 50:121-126.

Bashaw EC (1980). Apomixis and its application in crop improvement. In: Fehr WR and Hadley HH (ed) Hybridization of Crop Plants, American Society of Agronomy Crop Science Society of America Soil Science Society of America, Madison pp. 4563.

Broughton S (2008). Ovary co-culture improves embryo and green plant production in anther culture of Australian spring wheat (Triticum aestivum L.). Plant Cell Tissue and Organ Culture 95:185-195.

Caredda S, Doncoeur C, Devaux P, Sangwan RS, Clement C (2000). Plastid differentiation during androgenesis in albino and non-albino producing cultivars of barley (Hordeum vulgare L.). Sexual Plant Reproduction 13 (2): 95-104.

Chen J, Cui L, Malik AA, Mbira K (2011). In vitro haploid and dihaploid production via unfertilized ovule culture. Plant Cell Tissue and Organ Culture 104: 311-319.

Davies PA (2003). Barley isolated microspore culture (IMC) method. In: Maluszynski M, Kasha KJ, Forster BP, Szarejko I (eds.) Doubled haploid production in crop plants: A manual, Kluwer, Dordrecht, pp 49-52.

Davies P and Morton S (1998). A comparison of barley isolated microspore and anther culture and the influence of cell culture density. Plant Cell Reports 17: 206-210.

Devaux P and Kasha KJ (2009). Overview of barley doubled haploid production. In: Touraev A, Forster BP and Jain SM (eds.) Advances in Haploid Production in Higher Plants, Heidelberg, Springer: 4763.

Esteves P and Belzile FJ (2019). Isolated microspore culture in barley. In: Harwood W. (ed.) Barley: Methods in Molecular Biology, vol 1900. Humana Press, New York, NY.

Esteves P and Belzile F (2014a). Improving the efficiency of isolated microspore culture in six-row spring barley: I-optimization of key physical factors. Plant Cell Reports 33 (6): 993-1001.

Esteves P, Clermont I, Marchand S, Belzile F (2014b). Improving the efficiency of isolated microspore culture in six-row spring barley: II-exploring novel growth regulators to maximize embryogenesis and reduce albinism. Plant Cell Reports 33 (6): 871879.

Ferrie AMR and Caswell KL (2011). Isolated microspore culture techniques and recent progress for haploid and doubled haploid plant production. Plant Cell Tissue and Organ Culture 104: 301-309.

Ferrie A M R, Irmen KI, Beattie AD, Rossnagel BG (2014). Isolated microspore culture of oat (Avena sativa L.) for the production of doubled haploids: effect of pre-culture and post-culture conditions. Plant Cell Tissue and Organ Culture 116: 89-96.

Forster BP, Heberle-Bors E, Kasha KJ, Touraev A (2007). The resurgence of haploids in higher plants. Trends in Plant Science 12: 368-375. 
Foroughi-Wehr, B, Mix G, Gaul H and Wilson H M (1976). Plant production from cultured anthers of Hordeum vulgare. L. Z. Pflanzenzucht 77: 198-204.

Gaillard A, Vergne, P, Beckert M (1991). Optimization of maize microspore isolation and culture conditions for reliable plant regeneration. Plant Cell Reports 10: 5558.

Germana M A (2011). Anther culture for haploid and doubled haploid production. Trends Plant Science 104: 283-300.

Gland-Zwerger A, Javornik B, Bohanec B, Kreft I (1994). Production of doubled haploid lines through anther culture in barley and triticale. In: Javornik B and Bohanec B (eds.) Proceed. International Colloquium. Impact of Plant Biotechnology on Agriculture. December 5-7, Rogla, Slovenia: 9-14.

Hoekstra S, van Zijderveld $\mathrm{M} \mathrm{H}$, Louwerse J D, Heidekamp F, van der Mark F (1992). Anther and microspore culture of Hordeum vulgare L. cv Igri. Plant Science 86: 89-96.

Houben A, Sanei M, Pickering R (2011). Barley doubled-haploid production by uniparental chromosome elimination. Plant Cell Tissue and Organ Culture 104: 321-327.

$\mathrm{Hu}$ TC, Ziauddin A, Simion E, Kasha KJ (1995). Isolated microspore culture of wheat (Triticum aestivum L.) in a defined media. I. Effects of pretreatment, isolation methods, and hormones. In Vitro Cell and Developmental Biology 31: 79-83

Humphreys DG and Knox RE (2015). Doubled haploid breeding in cereals. In: Al-Khayri J, Jain S and Johnson D. (eds.) Advances in Plant Breeding Strategies: Breeding, Biotechnology and Molecular Tools, Springer, Cham.

Islam SSM, Ara I, Tuteja N, Subramaniam, S (2013). Efficient microspore isolation methods for high yield embryoids and regeneration in rice (Oryza sativa L.). International Journal of Biological Science and Engineering Vol: 7 No: 12.

Jacquard C, Wojnarowiez G, Clement C (2003). Anther culture in barley. In: Doubled haploid production in crop plants, Springer, Berlin, Heidelberg, Tokyo, pp. 21-28.

Jacquard C, Asakaviciute R, Hamalian AM, Sangwan R S, Devaux P, Clement C (2006). Barley anther culture: effects of annual cycle and spike position on microspore embryogenesis and albinism. Plant Cell Reports 25: 375-381.

Jahne A, Becker D, Brettschneider R, Lörz H. (1994). Regeneration of transgenic, microspore-derived, fertile barley. Theoretical and Applied Genetics 89 (4): 525-33.

Jansky S (2006). Overcoming hybridization barriers in potato. Plant Breeding 125 (1): 1-12.

Kasha KJ, Simion E, Miner M, Letarte J, Hu TC (2003). Haploid wheat isolated microspore culture protocol. In: Maluszynski M, Kasha KJ, Forster BP and Szarejko I (eds.) Doubled haploid production in crop plants: A manual, Kluwer, Dordrecht, pp 7781.

Köhler F and Wenzel G (1985). Regeneration of isolated barley microspores in conditioned media and trials to characterise the responsible factor. Journal of Plant Physiology 121: 181-191.

Letarte J, Simion E, Miner M, Kasha J (2006). Arabinogalactans and arabinogalactan-proteins induce embryogenesis in wheat (Triticum aestivum L.) microspore culture. Plant Cell Reports 24: 691.

Li H and Devaux P (2001). Enhancement of microspore culture efficiency of recalcitrant barley genotypes. Plant Cell Reports 20: 475-481.

Lippmann R, Friedel S, Mock HP, Kumlehn J (2015). The low molecular weight fraction of compounds released from immature wheat pistils supports barley pollen embryogenesis. Frontiers in Plant Science 6: 498.

Liu W, Zheng MY, Polle EA, Konzak CF (2002). Highly efficient doubled-haploid production in wheat Triticum aestivum L. via induced microspore embryogenesis. Crop Science 42: 686-692.

Makowska K, Oleszczuk S, Zimny J (2017). The effect of copper on plant regeneration in barley microspore culture. Czech Journal of Genetics and Plant Breeding 53 (1): 17-22.

Malayeri B E, Noori M, Jafari M (2012). Using the pollen viability and morphology for fluoride pollution biomonitoring. Biological Trace Element Research 147: 315-319.

Oleszczuk S, Sowa S, Zimny J (2006). Androgenic response to preculture stress in microspore cultures of barley. Protoplasma 228: 95-100.

Olsen FL (1991). Isolation and cultivation of embryogenic microspores from barley (Hordeum vulgare L.). Hereditas 115: 255-266.

Ouyang J W, Zhou SM, Jia SE (1993). The response of anther culture to culture temperature in Triticum aestivum. Theoretical and Applied Genetics 66: 101-109.

Patel M, Darvey NL, Marshall DR, Berry JO (2004). Optimization of culture conditions for improved plant regeneration efficiency from wheat microspore culture. Euphytica 140: 197-204.

Pechan PM and Keller WA (1989). Induction of microspore embryogenesis in Brassica napus L. by gamma irradiation and ethanol stress. In Vitro Cellular and Developmental Biology - Animal 25: 1073-1074.

Raina SK and Irfan ST (1998). High-frequency embryogenesis and plantlet regeneration from isolated microspores of indica rice. Plant Cell Reports 17: 957-962.

Rajcan I, Boersma JG, Shaw EJ (2011) Plant genetic techniques: Plant Breeder's Toolbox. Comprehen- 
sive Biotechnology (Second Edition), Volume 4. Elsevier.

Sestili S and Ficcadenti N (1996). Irradiated pollen for haploid production. In: Jain, SM, Sopory SK and Veilleux RE (eds.) In vitro haploid production in higher plants. Springer, Netherlands, pp. 263-274.

Shariatpanahi ME and Ahmadi B (2016). Isolated microspore culture and its applications in plant breeding and genetics. In: Anis M and Ahmad N (eds.) Plant Tissue Culture: Propagation, Conservation and Crop Improvement. Springer, Singapore.

Soriano M, Li H, Boutilier K (2013). Microspore embryogenesis: establishment of embryo identity and pattern in culture. Plant Reproduction 26: 181-196.

Tek A, Stupar R, Nagaki K (2015). Modification of centromere structure: a promising approach for haploid line production in plant breeding. Turkish Journal of Agriculture and Forestry 39 (4): 557562.

Torp AM and Andersen SB (2009). Albinism in microspore culture. In: Touraev A et al. (eds.) Advances in haploid production in higher plants, Springer, New York.

Vizintin L and Bohanec B (2004). In vitro manipulation of cucumber (Cucumis sativus L.) pollen and microspores: Isolation procedures, viability tests, germination, maturation. Acta Biologica Cracoviensia Series Botanica 46: 177-183.

Wang HM, Enns JL, Nelson KL (2019). Improving the efficiency of wheat microspore culture methodology: evaluation of pretreatments, gradients, and epigenetic chemicals. Plant Cell Tissue and Organ Culture 139: 589-599.

Wojnarowiez G, Caredda S, Devaux P, Sangwan R, Clement C (2004). Barley anther culture: assessment of carbohydrate effects on embryo yield, green plant production and differential plastid development in relation with albinism. Journal of Plant Physiology 161: 747-755.

Zhao JP, Simmonds DH, Newcomb W (1996). Induction of embryogenesis with colchicine instead of heat in microspores of Brassica napus L. cv. Topas. Planta 198: 433-439.

Zheng MY, Liu W, Weng Y, Polle E, Konzak CF (2001). Culture of freshly isolated wheat (Triticum aestivum L.) microspores treated with inducer chemicals. Plant Cell Reports 20: 685-690

Zheng M Y (2003). Microspore culture in wheat (Triticum aestivum) - doubled haploid production via induced embryogenesis. Plant Cell Tissue and Organ Culture 73: 213-230.

Ziauddin A, Simion E, Kasha KJ (1990). Improved plant regeneration from shed microspore culture in barley (Hordeum vulgare L.) cv. Igri. Plant Cell Reports 9: 69-72

Ziegler G, Dressler K, Hess D (1990). Investigations on the anther culturability of four German spring wheat cultivars and the influence of light on regeneration of green vs. albino plants. Plant Breeding 105: 40-46.

Zieliński K, Krzewska M, Żur I, Juzoń K, Kopeć P, Nowicka A, Moravčiková J, Skrzypek E, Dubas E (2020). The effect of glutathione and mannitol on androgenesis in anther and isolated microspore cultures of rye (Secale cereale L.). Plant Cell, Tissue and Organ Culture 140: 577-592.

Zur I, Dubas E, Krzewska M, Zieliński K, Fodor J, Janowiak F (2019). Glutathione provides antioxidative defence and promotes microspore-derived embryo development in isolated microspore cultures of triticale ( $\times$ Triticosecale Wittm.). Plant Cell Reports 38: 195-209. 